\title{
MAMMALS FROM THE TARASCAN PLATEAU, MICHOACÁN, MÉXICO
}

\author{
CONSTANTINO ORDUÑA TREJO ${ }^{1}$, ALONDRA CASTRO-CAMPILLO ${ }^{2}$, AND \\ JOSÉ RAMÍREZ-PULIDO ${ }^{2}$
}

\author{
1 Instituto Nacional de Investigaciones Forestales y Agropecuarias (INIFAP). \\ Campo Experimental Morelia, Michoacán, México. \\ ${ }^{2}$ Universidad Autónoma Metropolitana. Unidad Iztapalapa, División de C.B.S. \\ Departamento de Biología. Apartado Postal 55-535, Mexico 09340, D.F., México. \\ (Correo electrónico: jrp@xanum.uam.mx)
}

Resumen. Se examinaron 568 ejemplares de mamíferos nativos de 49 especies, procedentes de 49 localidades de la Meseta Tarasca o Sierra Purépecha del Estado de Michoacán, México. Las especies encontradas en esta región del Eje Volcánico Transversal incluyen 1 marsupial, 2 insectívoros, 17 quirópteros, 2 carnívoros, 24 roedores y 1 lagomorfo. Para calcular la diversidad de especies en los cuatro hábitats muestreados, se utilizaron las 2 especies de insectívoros y 21 de roedores. De mayor a menor diversidad, en el bosque de pino-encino se recolectaron 201 individuos de 15 especies $\left(H^{\prime}=2.49\right)$, en el bosque tropical caducifolio 142 de 15 especies $\left(H^{\prime}=2.43\right)$, en el bosque de oyamel 92 individuos de 10 especies $\left(H^{\prime}=2.01\right)$ y en las áreas de ecotono 52 individuos de cuatro especies $\left(H^{\prime}=1.15\right)$. El registro de Hylonycteris underwoodi es el primero para las partes altas del Estado de Michoacán.

Abstract. We examined 568 specimens of native mammals belonging to 47 species from 49 localities of the Tarascan Plateau or Sierra Purépecha mountain range in the State of Michoacán, México. Species composition of this enclave in the Transvolcanic Belt is 1 marsupial, 2 insectivores, 17 bats, 2 carnivores, 24 rodents, and 1 lagomorph. To calculate the species diversity according to the four sampled habitats, only the 2 insectivore and 21 rodent species were included. From higher to lower diversity values, we found 201 specimens from 15 species in pine-oak forest $\left(H^{\prime}=2.49\right), 142$ from 15 species in tropical deciduous forest $\left(H^{\prime}=2.43\right), 92$ specimens belonging to 10 species fir forest $\left(H^{\prime}=2.01\right)$, and 52 from four species in an ecotonal area $\left(H^{\prime}=\right.$ 1.15). Hylonycteris underwoodi is the first record for the highlands from the State of Michoacan.

Key words: Mammals, distribution, diversity, Tarascan Plateau, Michoacán. 


\section{INTRODUCTION}

Since the publication by Hall and Villa R. (1949), which referred to mammals from the north-central part of the State of Michoacán, only Burt (1961) and Orduña Trejo and Salas Páez (1993) have surveyed the region again. Other records for the region have appeared scattered in different papers dealing with mammals from the state (Huerta, 1989; Núñez-Garduño et al., 1996; Álvarez and Sánchez-Casas, 1997; González-Ruíz and Villalpando-R., 1997; Sánchez-Hernández et al., 1999). In fact, most mammal surveys from Michoacan have focused on the coastal region (Sánchez et al., 1985; 1992; Álvarez et al., 1987; Polaco and Muñiz-Martínez, 1987; Álvarez and Álvarez-Castañeda, 1991; Sánchez-Casas and Álvarez, 1997). The papers by Hooper (1961), and Núñez (1989) refer to the Sierra de Coalcomán. Additional papers mentioning mammals from the Transvolcanic Belt of the state are those by Williams and Ramírez-Pulido (1984); Rennert and Kilpatrick (1986), Houseal et al. (1987), Arita and Humphrey (1988), Tumlison (1991, 1992), Whitaker et al. (1991), Engstrom et al. (1992), Glendinning (1992), Alvarez and Hernández-Chávez (1993), Cervantes et al (1993) and, Castro-Campillo and Ramírez-Pulido (2000), among others.

Our objective here is to analyze the diversity of small mammals in three vegetation types (fir forest, pine-oak forest, and tropical deciduous forest) and ecotonal areas of the region known as Sierra Purépecha or Tarascan Plateau (Meseta Tarasca).

\section{MATERIALS AND METHODS}

\section{Study Area}

The Sierra Purépecha or Tarascan Plateau is located in the central-northwest part of the State of Michoacán, belonging to the Transvolcanic Belt. The area includes mountains and volcanos such as Paricutín, Tancítaro, Angahuan, San Marcos, and Comburindos. In that region, dominant vegetations are coniferous and oak forest with Pinus, Quercus, and Abies forests prevail, and at the boundary, tropical deciduous forest elements appear with genera such as Bursera, Acacia, Euphorbia (Rzedowski, 1978). The height of the Sierra ranges from $1,700 \mathrm{~m}$ to $3,200 \mathrm{~m}$.

Rainfall ranges from 1,250 to $1,650 \mathrm{~mm}$, and the climate is the most humid of the temperate subhumid ones with an annual mean temperature between 12 and $18^{\circ} \mathrm{C}$. Rainfall is concentrated in the summer, and winter rainfall is less than $5 \%$ of the total annual rainfall. Summers are cool and long, with mean temperature of the hottest month between 6.5 and $22^{\circ} \mathrm{C}$ (Gómez Tagle, 1984). 


\section{Plant communities}

The largest areas of fir forest occur in the mountainous region of the Tancítaro volcano, in the region of Angahuan-Zacán, 2,350 masl, and in Opopeo (2,100 masl) particularly in Cruz Gorda area in the Villa Escalante municipality. The fir forest in the central north region of the State of Michoacán is commonly found between 2,400 and a little over 3,000 masl. The fir forest consists of Abies religiosa associated with species of Pinus or Quercus (Bello González, 1983).

Pine-oak forests are widespread in the region. Representative three species include Pinus douglaciana, P. leiophylla, P. michoacana, P. pringlei, $P$. pseudostrobus, $P$. rudis, and $P$. teocote (Madrigal, 1982); oak species are represented by $Q$ uercus castanea, $Q$. candicans, $Q$. crassifolia, $Q$. crassipes, $Q$. glaucoiudes, $Q$. laurina, Q. magnoliifolia, Q. obtusata, Q. rugosa, and Q. scytophylla (Bello González and Labat, 1987). Other species associated with the pine-oak forest are Alnus arguta, A. firmifolia, Arbutus xalapensis, Carpinus caroliniana, Crataegus mexicana, Cletra mexicana, Cornus disciflora, Prunus capuli, Salix babilonica, Tilia mexicana, and Vitex mollis.

Transition forest, are located southwest of the City of Uruapan. Although, we could refer to this plant association as pine-oak forest also, it includes different species of pines and oak trees, at the ecotone with tropical deciduous forest. The prevailing species is Pinus occarpa, although P. michoacana is found in small areas. Several Quercus species such as $Q$. resinosa and $Q$. conspersa are also located there (Salas Paez, 1986).

The tropical deciduous forest is found in the northern part and in the northeast area of the Sierra Purépecha. The altitude limits are usually located between 1,500 and 2,000 masl. The annual mean temperature ranges between 17 and $21^{\circ} \mathrm{C}$, and rainfall ranges between 500 and $900 \mathrm{~mm}$ annually. Soils are sandy, acidic and poor in organic matter. The most common species are Bursera sp., Lysiloma acapulensis, Acacia penatula, A. farmesiana, A. angustissima, Ipomea murocoides, Opuntia sp., Cronton adsparsus, Agonandra racemosa, Lemaireocereus sp., Agave sp., Tecoma strans, Verbesina sphaero cephala, Bouvardia ternifolia, Euphorbia dentata, Tagetes lucida, Melapodium sp., Andropogon sp., Boutelova sp., Muhlenbergia sp., Senna huisuta, Paspalum sp., Mimosa monacista, and Eysenhardtia polystachya (Bello González, 1983).

\section{Field Work}

Fieldwork focused on the capture of rodents in areas within each major plant community. Sixteen trips were carried out and each plant community was sampled four times during three consecutive nights separated by a 45 -day interval. 
Bats were obtained by mist netting, at random sites in each vegetation type. For rodents and insectivorous, "Sherman" live-traps and "Museum Special" traps were used, with a mixture of oats and peanut butter as bait. The traps were placed on three lines in 20 stations, each one $20 \mathrm{~m}$ apart.

For medium sized mammals, "National" and "Tomahawk" live-traps were placed in a 20 -station line, each one $50 \mathrm{~m}$ apart. Canned sardine and/or pieces of fruit were used as bait. The presence of mammals was also detected by observing tracks and scats in a 30-minute transects (Rabinovich, 1984). Pocket gophers were trapped with "Volke" traps placed inside their tunnels, and checked during two consecutive days. All captured specimens were prepared as study skins and deposited in a collection of the Instituto Nacional de Investigaciones Forestales, Agrícolas y Pecuarias (INIFAP) at Uruapan, Michoacán.

Selection of sampling localities was made at random, with the traps placed in primary forest, and not in crop areas. Sampling localities appear on Figure 1, and the gazetteer in Appendix 1.

Diversity of mammals in plant communities was calculated with the ShannonWeaver's index, and the abundance was estimated using effort in traps/hour/night.

\section{RESULTS AND DISCUSSION}

We captured 568 specimens from 47 species including 1 marsupial, 2 insectivores, 17 bats, 2 carnivores, 24 rodents, and 1 lagomorph (Appendix 2). The former specific categories were obtained in 49 localities from the four habitats.

Capture rate in small mammals (excluding the three species of pocket gophers) was calculated including 487 specimens captured in 11,520 traps/hours/night. $18.8 \%$ were obtained from the fir forests, $41.5 \%$ from pine-oak forests, $29.0 \%$ from tropical deciduous forest and only $10.6 \%$ from the ecotone area (Fig. 2). Of the 23 species, 10 were found in the fir forest; 15 in the pine-oak forest; 15 in tropical deciduous forest and only four came from the ecotonal area (Table 1). Diversity was higher in the pineoak forest $\left(H^{\prime}=2.49\right)$, followed by the tropical deciduous forest $\left(H^{\prime}=2.43\right)$, fir forest $\left(H^{\prime}=2.01\right)$, and the ecotonal area $\left(H^{\prime}=1.15\right)$.

Highest diversity of mammals was found in pine-oak forest, followed by tropical deciduous forest. Although more individuals were captured in the pine-oak forest $(201)$, the diversity value $\left(H^{\prime}=2.49\right)$ was similar to the tropical deciduous forest $\left(H^{\prime}=2.43\right)$, where 142 specimens were trapped. In fact there were three dominant species in each one with Peromyscus gratus, P. maniculatus, and Reithrodontomys megalotis in the pine-oak forest representing $39.4 \%$ of the sample. Meanwhile, Liomys irroratus, Oligoryzomys fulvescens, and R. sumichrasti were dominant species in the tropical deciduous forest with $48.6 \%$ of the sample. Besides in 


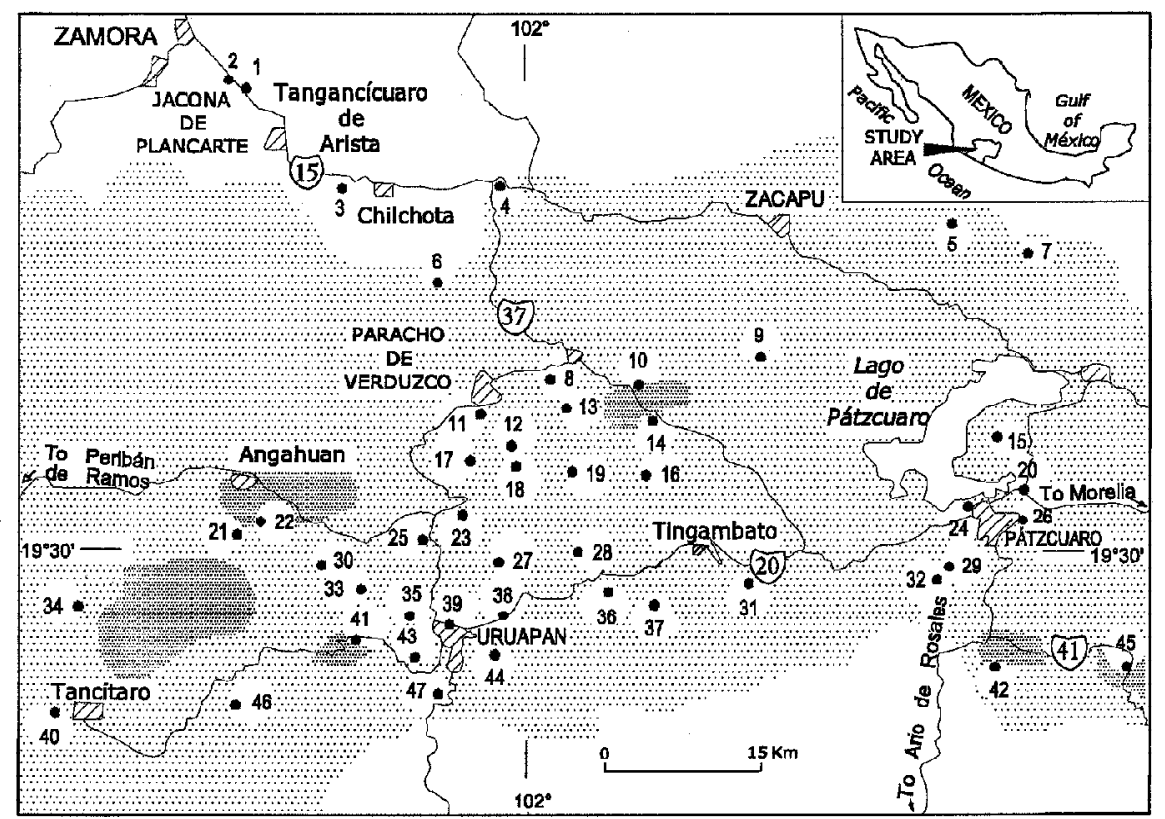

Figure 1. Regional map of the Tarascan Plateau, Michoacán, México. Numbers and dots correspond to gazetteer in Appendix 1. The vegetation types are indicated as follows: fir forest $=$ darkest areas, pine-oak forest $=$ doted areas, and tropical deciduous forest $=$ white areas.

the pine-oak forest, and in the tropical deciduous forest there were 12 additional species. Compared to the other two plant associations, these two habitats have highest diversity due to three shared species.

Species diversity diminishes in both fir forest and the ecotonal area. Diversity in the fir forest was lower since two species (Reithrodontomys megalotis, and $R$. sumichrasti) were the most abundant (47.8\%), and only other eight species occurred. Finally, in the ecotonal area, where the smallest diversity was obtained, there were only four species with 52 individuals and Peromyscus maniculatus represented $42.3 \%$ of the sample.

Nelsonia goldmani was captured only in a fir forest (Table 1). Peromyscus levipes, P. melanotis, and Sigmodon alleni were taken in the pine-oak forest. Cryptotis parva, Baiomys musculus, Neotoma mexicana, Oryzomys couesi, . melanophrys, $P$. spicilegus, and S. mascotensis were captured in the tropical deciduous forest. No species was restricted to the ecotonal area, and only P. aztecus, and Reithrodontomys 
megalotis appeared in all four habitats.

Sorex saussurei, Microtus mexicanus, and Neotomodon alstoni were captured in fir forest and pine-oak forest. Liomys irroratus, and Sigmodon hispidus were trapped in pine-oak forest, and tropical deciduous forest. Oligoryzomys fulvescens, $P$. gratus, and Reithrodontomys sumichrasti in fir forest, pine-oak forest, and tropical deciduous forest. Only $R$. chrysopsis was captured in fir forest, pine-oak forest, and ecotonal area.

The fir forest has one exclusive species; nine are shared with other plant associations, and 13 are absent. In the pine-oak forest, three species are exclusive, 12 are shared, and eight are absent. In the tropical deciduous forest seven are exclusive, eight are shared and eight are absent. In the ecotonal area, no species is exclusive, four are shared, and 19 are absent.

The mammals from the Tarascan Plateau or Sierra Purépecha mentioned in this paper represent one third of the total reported species for the State of Michoacán, which is one of the richest in México (Hall, 1981; Ramírez-Pulido et al., 1986;

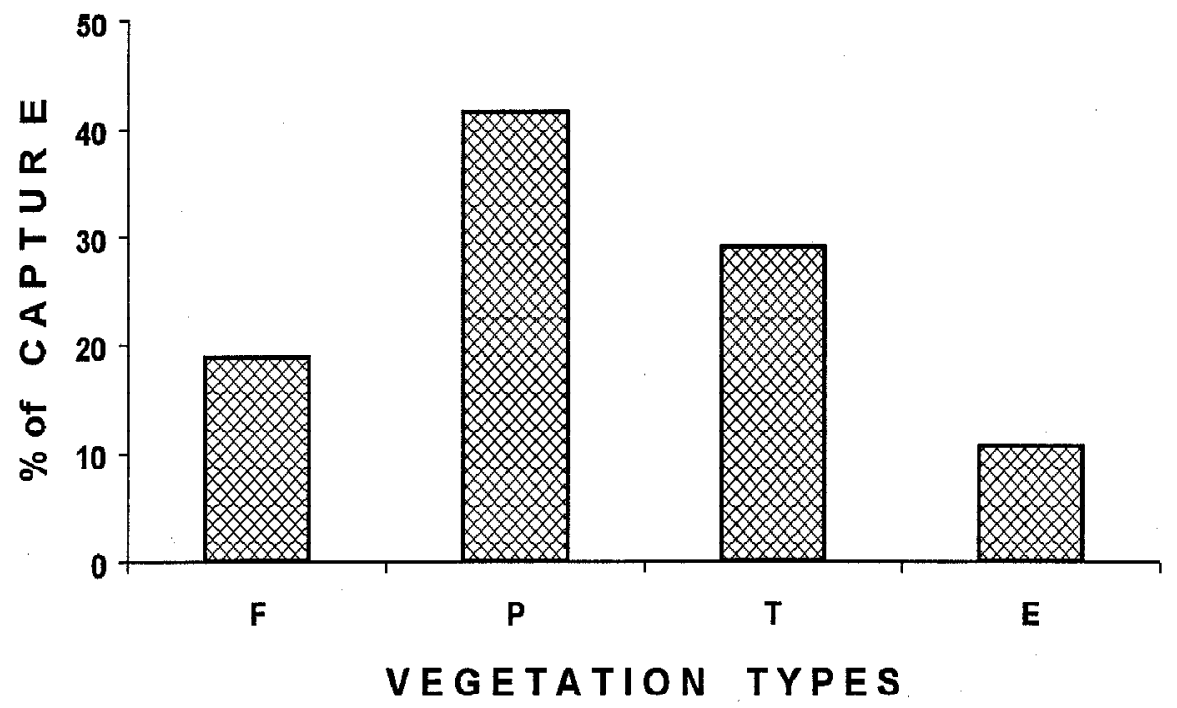

Figure 2. Percentage of capture of rodent and insectivore species in four plant communities at the Tarascan Plateau, Michoacán. $\mathrm{F}=$ fir forest, $\mathrm{P}=$ pine-oak forest, $\mathrm{T}=$ tropical deciduous forest, and $\mathrm{E}=$ ecotonal area. 
Table 1. Insectivores and rodents collected in four types of vegetation in the Tarascan Plateau, Michoacán, México. $\mathrm{F}$ = fir forest; $\mathrm{P}=$ pine-oak forest; $\mathrm{T}=$ tropical deciduous forest; and $\mathrm{E}=$ ecotonal area.

\begin{tabular}{|c|c|c|c|c|}
\hline S P E C I E S & $\mathrm{F}$ & $\mathrm{P}$ & $\mathrm{T}$ & $\mathrm{E}$ \\
\hline Cryptotis parva & 0 & 0 & 1 & 0 \\
\hline Sorex saussurei & 2 & 3 & 0 & 0 \\
\hline Liomys irroratus & 0 & 4 & 22 & 0 \\
\hline Microtus mexicanus & 11 & 19 & 0 & 0 \\
\hline Baiomys musculus & 0 & 0 & 8 & 0 \\
\hline Nelsonia goldmani & 2 & 0 & 0 & 0 \\
\hline Neotoma mexicana & 0 & 0 & 4 & 0 \\
\hline Neotomodon alstoni & 12 & 6 & 0 & 0 \\
\hline Oligoryzomys fulvescens & 7 & 7 & 25 & 0 \\
\hline Oryzomys couesi & 0 & 0 & 1 & 0 \\
\hline Peromyscus hylocetes & 6 & 18 & 7 & 1 \\
\hline Peromyscus gratus & 5 & 33 & 8 & 0 \\
\hline Peromyscus levipes & 0 & 15 & 0 & 0 \\
\hline Permyscus maniculatus & 0 & 20 & 11 & 22 \\
\hline Peromyscus melanotis & 0 & 7 & 0 & 0 \\
\hline Peromyscus melanophrys & 0 & 0 & 7 & 0 \\
\hline Peromyscus spicilegus & 0 & 0 & 5 & 0 \\
\hline Reithrodontomys chrysopsis & 3 & 6 & 0 & 13 \\
\hline Reithrodontomys megalotis & 20 & 27 & 6 & 16 \\
\hline Reithrodontomys sumichrasti & 24 & 17 & 22 & 0 \\
\hline Sigmodon alleni & 0 & 3 & 0 & 0 \\
\hline Sigmodon hispidus & 0 & 16 & 9 & 0 \\
\hline Sigmodon mascotensis & 0 & 0 & 6 & 0 \\
\hline TO T A L & 92 & 201 & 142 & 52 \\
\hline
\end{tabular}

Ramírez-Pulido and Castro-Campillo, 1990; 1993; 1994a; 1994b).

The three specimens of Hylonycteris underwoodi from the neighborhood of Uruapan are noteworthy since they represent the first record for the species at 1,600 masl in the Tarascan Plateau. This species was firstly recorded for the state in the Coastal Region between 800 masl and 980 masl (Polaco and Muñíz-Martínez, 1987). 


\section{ACKNOWLEDGMENTS}

Dr. Miguel Angel Armella provided useful suggestions to an earlier version of the manuscript. We are particularly grateful to Dr. Guy G. Musser of the American Museum of Natural History, whose comments and critical remarks enriched the paper. The first draft of figure 1 was drawn by Mr. Fernando Vega, and the final edition by Miss Diana I. López. We gratefully acknowledge their promptness in attending our countless requests. We thank an anonymous reviewer for his criticisms that enhanced the final version of the manuscript. This project was partially financed by the Consejo Nacional de Ciencia y Tecnología (No. 400200-5R29117N).

\section{LITERATURE CITED}

Álvarez, T. y S. T. Álvarez-Castañeda. 1991. Notas sobre el estado taxonómico de Pteronotus davyi en Chiapas y de Hylonycteris en México (Mammalia: Chiroptera). Anales de la Escuela Nacional de Ciencias Biológicas, México, 34: 223-229.

Álvarez, T., J. Arroyo-Cabrales y M. González-Escamilla. 1987. Mamíferos (excepto Chiroptera de la costa de Michoacán, México). Anales de la Escuela Nacional de Ciencias Biológicas, México, 31: 13-62.

Álvarez, T. y J. J. Hernández-Chávez. 1993. Taxonomía del metorito Microtus mexicanus en el centro de México con la descripción de una nueva subespecie. Pp. 137-156 en, Avances en el estudio de los mamíferos de México (R. A. Medellín y G. Ceballos, eds). Asociación Mexicana de Mastozoología, Publicaciones Especiales, 1: 1-464.

Álvarez, T. y N. Sánchez-Casas. 1997. Contribución al conocimiento de los mamíferos, excepto Chiroptera y Rodentia, de Michoacán, México. Anales de la Escuela Nacional de Ciencias Biológicas, México, 42: 47-74.

Arita, H. y S. R. Humphrey. 1988. Revisión taxonómica de los murciélagos magueyeros del género Leptonycteris (Chiroptera: Phyllostomidae). Acta Zoológica Mexicana, nueva serie, 29: 1-60.

Bello González, M. A. 1983. Estudio fenológico de 5 especies de Pinus en la región de Uruapan, Mich. (México). Tesis Profesional, Universidad Nacional Autónoma de México.

Bello González, M. A. y J.N. Labat. 1987. Los encinos (Quercus) del Estado de Michoacán, México. Coedición de la Secretaría de Agricultura y Recursos Hidráulicos, Instituto Nacional de Investigaciones Forestales y Agropecuarias y Centre D'Etudes Mexicaines et Centramericaines. Collection Etudes Mesoaméricanes, Serie 11-9, Cuadernos de Estudios Michoacanos 1.

Burt, W. H. 1961. Some effects of volcan Paricutin on vertebrates. Occasional Papers of the Museum of Zoology, University of Michigan, 620: 1-24.

Castro-Campillo, A. and J. Ramírez-Pulido. 2000. Systematics of the smooth-toothed gopher, Thomomys umbrinus, in the Mexican Transvolcanic Belt. American Museum Novitates, 
329:1-37.

Cervantes, F. A., V. J. Sosa, J. Martínez, R. Ma. González, and R. J. Dowler. 1993. Pappogeomys tylorhinus. Mammalian Species, 433: 1-4.

Engstrom, M. D., O. Sánchez-Herrera, and G. Urbano-Vidales. 1992. Distribution, geographic variation, and systematic relationships within Nelsonia (Rodentia: Sigmodontinae). Proceedings of the Biological Society of Washington, 105: 867-881.

García, E. 1981. Modificación climática de Köppen. Universidad Nacional Autónoma de México, México, D.F.

Glendinning, J. I. 1992. Range extension for the diminutive woodrat, Nelsonia neotomodon, in the Mexican Transvolcanic range. Southwestern Naturalist, 37: 92-93.

Gómez Tagle, R. A. 1984. Condiciones generales de los recursos naturales en la Sierra Purépecha, Mich. Memoria. Primer encuentro Purépecha sobre el manejo tradicional de los recursos naturales. Secretaría de Desarrollo Urbano y Ecología, Secretaría de Educación Pública, Instituto Nacional de Investigaciones Forestales y Gobierno de Michoacán.

González-Ruíz, N. y J. Villalpando-R. 1997. Primer registro de murciélagos y segundo de Myotis auriculus apache (Mammalia: Chiroptera) para Michoacán, México. Vertebrata Mexicana, 4: 13-16.

Hall, E. R. 1981. The mammals of North America. John Wiley and Sons, vol. 1: XV+600+90, vol. 2: VI+601-1181+90.

Hall, E. R., and B. Villa-R. 1949. An annotated checklist of the mammals of Michoacan, Mexico. University of Kansas Publications, Museum of Natural History, 1: 431-472.

Hamilton, W. J., Jr., and J. O. Whitaker, Jr. 1979. Mammals of the Eastern United States. Second Edition, Cornell University Press, EUA.

Hooper, E. T. 1961 Notes on mammals from western and southern Mexico. Journal of Mammalogy, 42: 120-122.

Houseal, T. W., I. F. Greenbaum, D. J. Schmidly, S. A. Smith, and K. M. Davis. 1987. Karyotypic variation in Peromyscus boylii from Mexico. Journal of Mammalogy, 68: 281-296.

Huerta, M. C. 1989. Nuevos registros de murciélagos para el Estado de Michoacán. Boletín de la Coordinación de Investigación Científica, Universidad Michoacana San Nicolás de Hidalgo, 13: 38-39.

Madrigal, S. X. 1982. Clave para la identificación de las coníferas silvestres del Estado de Michoacán. Boletín Divulgativo Instituto Nacional de Investigaciones Forestales, México, 58: $1-100$.

Núñez G., A. 1989. Los mamíferos silvestres de Michoacán. Boletín de la Coordinación de Investigación Científica, Universidad Michoacana San Nicolás de Hidalgo, 12: 22-26.

Núñez-Garduño, A., C. Sánchez-Hernández, and Ma. de L. Romero-Almaraz. 1996. Noteworthy records of some bats from Michoacán, México. Bat Research News, 37: 39-40.

Orduña Trejo, C. y M. A. Salas Páez. 1993. Los mamíferos de la Sierra Purépecha del Estado de Michoacán. Boletín Divulgativo. Secretaría de Agricultura y Recursos Hidraúlicos, Instituto Nacional de Investigaciones Forestales y Agropecuarias, División Forestal, 78: 1-31.

Polaco, O. J. y R. Muñiz-Martínez. 1987. Los murciélagos de la costa de Michoacán. Anales de la Escuela Nacional de Ciencias Biológicas, México, 31: 68-89. 
Rabinovich, J. E. 1984. Introducción a la ecología de poblaciones animales. Edit. Continental, S.A.

Ramírez-Pulido, J., M. C. Britton, A. Perdomo y A. Castro. 1986. Guía de los mamíferos de México. Referencias hasta 1983. Ediciones de la Universidad Autónoma Metropolitana, Iztapalapa, México, D.F.

Ramírez-Pulido, J. y A. Castro-Campillo. 1990. Bibliografía reciente de los mamíferos de México, 1983-1988. Colección CBS. Ediciones de la Universidad Autónoma Metropolitana, Iztapalapa, México, D.F.

Ramírez-Pulido, J. y A. Castro-Campillo. 1993. Diversidad mastozoológica en México. Pp. 413-427 en, Diversidad biológica en México (R. Gío-Argáez y E. López-Ochoterena, eds.). Revista de la Sociedad Mexicana de Historia Natural, Volumen Especial, 44: 1-427.

Ramírez-Pulido, J. y A. Castro-Campillo. 1994a. Bibliografía reciente de los mamíferos de México, 1989-1993. Ediciones de la Universidad Autónoma Metropolitana, Iztapalapa, México, D.F.

Ramírez-Pulido, J. y A. Castro-Campillo. 1994b. Análisis multivariado de los mamíferos mexicanos a nivel estatal con una modificación al algoritmo de Peters. Revista de la Sociedad Mexicana de Historia Natural, 45: 61-74.

Rennert, P. D., and C. W. Kilpatrick. 1986. Biochemical systematics of populatios of Peromyscus boylii. I. Populations from east-central Mexico with low fundamental numbers. Journal of Mammalogy, 67: 481-488.

Rzedowski, J. 1978. Vegetación de México. Ed. Limusa, S. A.

Salas Páez, M. A. 1986. Aves de la Sierra Purépecha, Estado de Michoacán. Tesis Profesional, Universidad Nacional Autónoma de México.

Sánchez-Casas, N. y T. Álvarez. 1997. Notas sobre la dieta de Hylonycteris (Chiroptera: Phyllostomidae) en México. Vertebrata Mexicana, 3: 9-12.

Sánchez H., C., Ma. de L. Romero A. y A. Núñez G. 1992. El oso hormiguero Tamandua mexicana en la costa del Estado de Michoacán. Southwestern Naturalist, 37: 88-89.

Sánchez-Hernández, C., C. B. Chávez-Tapia, A Núñez-Garduño, E. Ceballos-Corona, and M. A. Gurrola-Hidalgo. 1985. Notes on distribution and reproduction of bats from coastal regions of Michoacan, Mexico. Journal of Mammalogy, 66: 549-553.

Sánchez-Hernández, C., Ma. de L. Romero-Almaraz, R. D. Owen, A. Núñez-Garduño, and R. López-Wilchis. 1999. Noteworthy records of mammals from Michoacán, México. Southwestern Naturalist, 44: 231-235.

Tumlison, R. 1991. Bats of the genus Plecotus in Mexico: discrimination and distribution. Occasional Papers of the Museum, Texas Tech University, 140: 1-19.

Tumlison, R. 1992. Plecotus mexicanus. Mammalian Species, 401: 1-3.

Whitaker, J. O., Jr., J. L. Glendinning, and W. J. Wrenn. 1991. Ectoparasites of Sorex saussurei (Saussure's shrew) from Michoacan, Mexico. Southwestern Naturalist, 35: 114115.

Williams, S. L., and J. Ramírez-Pulido. 1984. Morphometric variation in the volcano mouse Peromyscus (Neotomodon) alstoni (Mammalia: Cricetidae) Annals of Carnegie Museum, 53:163-183. 
Appendix 1. Gazetteer of localities in the Tarascan Plateau, Michoacán.

1. $5 \mathrm{Km}$ SE Zamora, 1,800 masl

2. $4 \mathrm{Km}$ SE Zamora, 1,800 masl

3. Los Nogales, 1,750 masl

4. San Antonio Carapan, 2,000 masl

5. La Naranja, $15 \mathrm{Km}$ E Zacapu, 2,000 masl

6. Tanaco, 2,200 masl

7. Cerro Gacho, 2,600 masl

8. Cherán, 2,200 masl

9. Cerro de la Mojonera, 3,000 masl

10. Nahuatzen, 2,800 masl

11. Paracho, 2,750 masl

12. $6 \mathrm{Km}$ SE Paracho, 2,800 masl

13. Cerro San Marcos, 2,900 masl

14. Sevina, 2,600 masl

15. Cerro del Timbén, Mabusco, 2,100 masl

16. Cerro de la Bandera, Comachuén, 2,900 masl

17. $8 \mathrm{Km} \mathrm{N}$ Capacuaro, 2,600 masl

18. $10 \mathrm{Km}$ NE Capacuaro, 2,500 masl

19. Cerro Cicopién, 2,600 masl

20. Tzurumútaro, 2,100 masl

21. Cerro Los Gallineros, $2.5 \mathrm{Km} \mathrm{SW}$ Angahuan, 2,400 masl $19^{\circ}$

22. $2 \mathrm{Km}$ SE Angahuan, 2,450 masl

23. Cerro del Banco, Capacuaro, 2,350 masl

24. Bonilla, 2,100 masl

25. San Lorenzo, $17 \mathrm{Km}$ NE Uruapan, 1,900 masl

26. Pátzcuaro, 2,500 masl

27. El Columpio, Pátzcuaro, 2,500 masl

27. Teamba, 1,900 masl

28. Montelongos, 2,000 masl

29. $12 \mathrm{Km} \mathrm{N}$ Villa Escalante, 2,500 masl

30. Cerro La Mejorana, 2,200 masl

31. Cerro Comburindos, Tingambato, 2,350 masl

32. $9 \mathrm{Km}$ SW Pátzcuaro, 1,900 masl

33. Cerro Las Cocinas, 2,250 masl

34. Derramadero, $12 \mathrm{Km}$ NW Tancítaro, 2,200 masl

35. $5 \mathrm{Km} \mathrm{NW}$ Uruapan, 2,650 masl

36. $6 \mathrm{Km} \mathrm{N}, 15 \mathrm{Km}$ E Uruapan, 1,850 masl

37. San Angel Zurumucapio, 1,700 masl

38. Santa Catarina, 1,600 masl

39. Barranca del Cupatitzio, 1,640 masl $19^{\circ} 56^{\prime} 05^{\prime \prime}, 102^{\circ} 15^{\prime} 11^{\prime \prime}$

$19^{\circ} 56^{\prime} 30^{\prime \prime}, 102^{\circ} 15^{\prime} 58^{\prime \prime}$

$19^{\circ} 50$ ' 10 ', $102^{\circ} 09^{\prime} 53^{\prime \prime}$

$19^{\circ} 50^{\prime} 32^{\prime \prime}, 102^{\circ} 02^{\prime} 09^{\prime \prime}$

$19^{\circ} 48^{\prime} 04^{\prime \prime}, 101^{\circ} 39^{\prime} 23^{\prime \prime}$

$19^{\circ} 43^{\prime} 04^{\prime \prime}, 102^{\circ} 05^{\prime} 28^{\prime \prime}$

$19^{\circ} 46^{\prime} 30^{\prime \prime}, 101^{\circ} 36^{\prime} 32^{\prime \prime}$

$19^{\circ} 39^{\prime} 45^{\prime}, 101^{\circ} 58^{\prime} 07^{\prime \prime}$

$19^{\circ} 40^{\prime} 32^{\prime}, 101^{\circ} 50^{\prime} 03$ "

$19^{\circ} 38^{\prime} 00^{\prime \prime}, 101^{\circ} 55^{\prime} 11^{\prime \prime}$

$19^{\circ} 37^{\prime} 35^{\prime}, 102^{\circ} 03^{\prime} 02$ "

$19^{\circ} 34^{\prime}$ '56", $102^{\circ} 00^{\prime} 46^{\prime \prime}$

$19^{\circ} 38^{\prime} 24^{\prime \prime}, 101^{\circ} 58^{\prime} 50^{\prime \prime}$

$19^{\circ} 36^{\prime}$ '52", $101^{\circ} 54^{\prime} 41^{\prime}$ "

$19^{\circ} 36^{\prime} 13^{\prime \prime}, 101^{\circ} 37^{\prime} 05^{\prime \prime}$

$19^{\circ} 32^{\prime} 50^{\prime \prime}, 101^{\circ} 55^{\prime} 05^{\prime \prime}$

$19^{\circ} 36^{\prime} 26^{\prime \prime}, 102^{\circ} 03$ ' $48^{\prime \prime}$

$19^{\circ} 35^{\prime} 00^{\prime \prime}, 101^{\circ} 59^{\prime} 30^{\prime \prime}$

$19^{\circ} 33^{\prime} 30^{\prime \prime}, 101^{\circ} 58^{\prime} 23$ "

$19^{\circ} 32^{\prime} 17^{\prime \prime}, 101^{\circ} 35^{\prime} 34^{\prime \prime}$

$19^{\circ} 30^{\prime} 43^{\prime \prime}, 102^{\circ} 15^{\prime} 08^{\prime \prime}$

$19^{\circ} 31^{\prime} 26^{\prime}, 102^{\circ} 13^{\prime} 02^{\prime \prime}$

$19^{\circ} 31^{\prime} 52^{\prime}, 102^{\circ} 03^{\prime} 48^{\prime}$ "

$19^{\circ} 31^{\prime} 30^{\prime \prime}, 101^{\circ} 42^{\prime} 16^{\prime \prime}$

$19^{\circ} 30^{\prime} 21^{\prime \prime}, 102^{\circ} 07^{\prime} 37^{\prime \prime}$

$19^{\circ} 30^{\prime} 43^{\prime \prime}, 102^{\circ} 36^{\prime} 30^{\prime \prime}$

$19^{\circ} 30^{\prime} 43^{\prime \prime}, 102^{\circ} 36^{\prime} 30^{\prime \prime}$

$19^{\circ} 29^{\prime} 31^{\prime \prime}, 102^{\circ} 01^{\prime} 52^{\prime \prime}$

$19^{\circ} 29^{\prime} 39^{\prime \prime}, 101^{\circ} 56^{\prime} 18^{\prime \prime}$

$19^{\circ} 28^{\prime} 00^{\prime \prime}, 101^{\circ} 39^{\prime} 02^{\prime \prime}$

$19^{\circ} 28^{\prime} 40^{\prime \prime}, 102^{\circ} 09^{\prime} 26^{\prime \prime}$

$19^{\circ} 29^{\prime} 56^{\prime}, 101^{\circ} 51^{\prime} 16^{\prime}$ "

$19^{\circ} 27^{\prime} 17^{\prime}, 101^{\circ} 40^{\prime} 25^{\prime \prime}$

$19^{\circ} 27^{\prime} 27^{\prime \prime}, 102^{\circ} 07^{\prime} 04^{\prime \prime}$

$19^{\circ} 27^{\prime} 13^{\prime}, 102^{\circ} 21^{\prime} 13^{\circ}$ "

$19^{\circ} 26^{\prime} 08^{\prime \prime}, 102^{\circ} 06^{\prime} 21^{\prime \prime}$

$19^{\circ} 27^{\prime} 17^{\prime}, 102^{\circ} 55^{\prime} 58^{\prime \prime}$

$19^{\circ} 27^{\prime} 39^{\prime \prime}, 101^{\circ} 54^{\prime} 35^{\prime \prime}$

$19^{\circ} 26^{\prime} 17^{\prime \prime}, 102^{\circ} 01^{\prime} 30^{\prime \prime}$

$19^{\circ} 25^{\prime} 50^{\prime \prime}, 102^{\circ} 03^{\prime} 06^{\prime}$ " 
Appendix 1. Cont. ...

39. Campo Experimental Forestal del Cupatitzio, 1640 masl

40. $1 \mathrm{Km} \mathrm{N}, 2 \mathrm{Km}$ W Tancítaro, 2,900 masl

41. San Juan Nuevo, 1,850 masl

42. El Abrojal, Opopeo, 2,100 masl

43. $4 \mathrm{Km} \mathrm{SW}$ Uruapan, 1,760 masl

44. La Huizachera, Santa Rosa, 1,600 masl

45. 2 km W Cruz Gorda, 3,100 masl

46. Los Barrancos Cutzato, 1,700 masl

47. Presa del Cupatitzio, 1,600 masl $19^{\circ} 25^{\prime} 50^{\prime \prime}, 102^{\circ} 03^{\prime} 06^{\prime \prime}$

$19^{\circ} 25^{\prime} 21^{\prime \prime}, 102^{\circ} 16^{\prime} 05^{\prime \prime}$

$19^{\circ} 24^{\prime} 13$ ", $102^{\circ} 08^{\prime} 13^{\prime \prime}$

$19^{\circ} 24^{\prime} 34^{\prime \prime}, 101^{\circ} 36^{\prime} 27^{\prime \prime}$

$19^{\circ} 22^{\prime} 39$ ', $102^{\circ} 05^{\prime} 58^{\prime \prime}$,

$19^{\circ} 24^{\prime} 05^{\prime}, 102^{\circ} 02^{\prime} 35^{\prime}$ "

$19^{\circ} 24^{\prime} 12^{\prime \prime}, 101^{\circ} 29^{\prime} 30^{\prime \prime}$

$19^{\circ} 20^{\prime} 54$ ', $102^{\circ} 15^{\prime} 28^{\prime \prime}$

$19^{\circ} 24^{\prime} 01^{\prime \prime}, 102^{\circ} 05^{\prime} 00^{\prime}$ " 
Appendix 2. Specimens examined.

Didelphis marsupialis caucae J. A. Allen, 1900. Specimens examined: Barranca del Cupatitzio, 1,640 masl (1).

Cryptotis parva berlandieri (Baird, 1858). Specimens examined: Tzurumútaro, 2,100 masl (1).

Sorex saussurei saussurei Merriam, 1892. Specimens examined: Cerro los Gallineros, Angahuan, 2,400 masl (2); Cerro Comburindos, Tingambato, 2,350 masl (1); El Abrojal, Opopeo, 2,100 masl (2);

Pteronotus parnellii mexicanus (Miller, 1902). Specimen examined: La Huizachera, Santa Rosa, 1,600 masl (1).

Macrotus waterhousii mexicanus Saussure, 1860. Specimens examined: $10 \mathrm{Km}$ NE Capacuaro, 2,500 masl (1); $5 \mathrm{Km}$ NW Uruapan, 2,650 masl (1).

Desmodus rotundus murinus Wagner, 1840. Specimen examined: 5 Km NW Uruapan, 2,650 masl (1); Barranca del Cupatitzio, 1,640 masl (1).

Anoura geoffroyi lasiopyga (Peters, 1868). Specimen examined: Cerro Las Cocinas, 2,250 masl (1); Tiamba, 1,900 masl (1); Barranca del Cupatitzio, 1,640 masl (1).

Choeronycteris mexicana Tschudi, 1844. Specimen examined: Barranca del Cupatitzio, 1,640 masl (1).

Glossophaga soricina handleyi Webster y Jones, 1980. Specimen examined: $10 \mathrm{Km} \mathrm{NE}$ Capacuaro, 2,500 masl (1).

Hylonycteris underwoodi minor Phillips y Jones, 1971. Specimen examined: La Huizachera, Santa Rosa, 1,600 masl (1); Presa del Cupatitzio, 1,600 masl (2).

Leptonycteris nivalis (Saussure, 1460). Specimens examined: $10 \mathrm{Km}$ NE Capacuaro, 2,500 masl (1); Campo Experimental Forestal del Cupatitzio, Uruapan, 1,640 masl (1); Barranca del Cupatitzio, 1,640 masl (4).

Artibeus jamaicensis triomylus Handley, 1966. Specimen examined: 5 Km NW Uruapan, 2,650 masl (1).

Chiroderma salvini scopaeum Handley, 1966. Specimen examined: Cerro del Banco, Capacuaro, 2,350 masl (1).

Dermanura azteca azteca (Andersen, 1906). Specimens examined: Tiamba, 1900 masl (1); San Lorenzo, 17 Km NE Uruapan, 1900 masl (2); San Angel Zurumucapio, 1,700 masl (2).

Sturnira ludovici ludovici Anthony, 1924. Specimens examined: 2 Km SW Angahuan, 2290 masl (2); 5 Km NW Uruapan, 2,650 masl (9); Carretera Tancítaro, Santa Catarina, 1,600 masl (1); San Angel Zurumucapio, 1700 masl (11).

Corynorhinus mexicanus G. MASL. Allen, 1916. Specimen examined: 5 Km NW Uruapan, 2,650 masl (1); El Abrojal, Opopeo, 2,100 masl (1).

Eptesicus fuscus miradorensis (H. Allen, 1866). Specimen examined: Campo Experimental Forestal del Cupatitzio, Uruapan, 1,640 masl (11). 
Myotis californica mexicana (Saussure, 1860). Specimen examined: Barranca del Cupatitzio, 1,640 masl (1).

Appendix 2. Cont. ...

Myotis thysanodes thysanodes Miller, 1897. Specimen examined: Barranca del Cupatitzio, 1,640 masl (3).

Myotis velifera velifera (J. A. Allen, 1890). Specimen examined: Campo Experimental Forestal del Cupatitzio, Uruapan, 1,640 masl (1).

Mephitis macroura macroura Lichtenstein, 1832. Specimen examined: Barranca del Cupatitzio, 1,640 masl (1).

Mustela frenata leucoparia (Merriam, 1896). Specimen examined: El Abrojal, Opopeo, 2,100 masl (1).

Cratogeomys gymnurus imparilis (Goldman, 1939). Specimens examined: Nahuatzen, 2,800 masl (1); San Lorenzo, 17 Km NE Uruapan, 1900 masl (1); El Abrojal, Opopeo, 2,100 masl (1).

Cratogeomys tylorhinus angustirostris (Merriam, 1903). Specimen examined: Derramadero, 12 Km NW Tancítaro, 2,200 masl (1); Barranca del Cupatitzio, 1,640 masl (2).

Zygogeomys trichopus trichopus Merriam, 1895. Specimens examined: Nahuatzen, 2,800 masl (2); Barranca del Cupatitzio, 1,640 masl (2).

Liomys irroratus alleni (Coues, 1881). Specimens examined: Los Nogales, 1,750 masl (4); La Naranja, $15 \mathrm{Km}$ E Zacapu, 2,000 masl (5); Cerro Comburindos, Tingambato, 2,350 masl (4); Bonilla, 2,100 masl (1); Pátzcuaro, 2,500 masl (6); El Columpio, Pátzcuaro, 2,500 masl (4); 9 Km SW Pátzcuaro, 1900 masl (2).

Microtus mexicanus fundatus Hall, 1948. Specimens examined: Cerro de la Mejorana, 2,200 masl (2); Sevina, 2,600 masl (8); $2 \mathrm{Km} \mathrm{W}$ Cruz Gorda, 2,300 masl (3); $2 \mathrm{Km} \mathrm{SE}$ Angahuan, 2,450 masl (9); Cerro Los Gallineros, 2.5 Km SW Angahuan, 2,400 masl (3); $12 \mathrm{Km}$ N Villa Escalante, 2,500 masl (1); El Abrojal, Opopeo, 2,100 masl (4).

Baiomys musculus pallidus Russell, 1952. Specimens examined: Los Nogales, 1,750 masl (1); Cerro Las Cocinas, 2,250 masl (1); 9 Km SW Pátzcuaro, 1900 masl (4); El Columpio, Pátzcuaro, 2,500 masl (1); Tzurumútaro, 2,100 masl (1).

Nelsonia goldmani goldmani Merriam, 1903. Specimens examined: Cerro San Marcos, 2,900 masl (2).

Neotoma mexicana tenuicauda Merriam, 1892. Specimens examined: Los Nogales, 1,750 masl (1); Bonilla, 2,100 masl (1); Pátzcuaro, 2,500 masl (1); Derramadero, 12 Km NW Tancítaro, 2,200 masl (1).

Neotomodon alstoni Merriam, 1898. Specimens examined: Cerro San Marcos, 2,900 masl, (9); El Abrojal, Opopeo, 2,100 masl (7); 2 Km W Cruz Gorda, 3100 masl (2).

Oligoryzomys fulvescens lenis (Goldman, 1915). Specimens examined: Los Nogales, 1,750 masl (8); Cerro San Marcos, 2,900 masl (4); Sevina, 2,600 masl (1); Cerro Cicopién, 2,600 masl (1); Bonilla, 2,100 masl (3); $6 \mathrm{Km} \mathrm{N}, 15 \mathrm{Km}$ E Uruapan, 1,850 masl (3); Montelongos, 2,000 masl (1); Pátzcuaro, 2,500 masl (6); $9 \mathrm{Km} \mathrm{SW,} \mathrm{Pátzcuaro,} \mathrm{1,900} \mathrm{masl} \mathrm{(1);} \mathrm{El}$ 
Abrojal, Opopeo, 2,100 masl (2); San Juan Nuevo, 1850 masl (1); La Huizachera, Santa Rosa, 1,600 masl (2); Barranca del Cupatitzio, 1,640 masl (2); 2 Km W Cruz Gorda, 3100 masl (4).

Appendix 2. Cont. ...

Oryzomys couesi regillus Goldman, 1915. Specimens examined: $3 \mathrm{Km}$ W Venustiano Carranza (not shown in map) (1).

Peromyscus hylocetes Merriam, 1897. Specimens examined: 8 Km N Capacuaro, 2,600 masl (3); Cerro de la Bandera, Comachuén, 2,900 masl (1); 6 Km N, 15 Km E Uruapan, 1,850 masl (19); $2 \mathrm{Km}$ W Cruz Gorda, 3100 masl (2); Cerro Comburindos, Tingambato, 2,350 masl (1); El Abrojal, Opopeo, 2,100 masl, (1); Pátzcuaro, 2,500 masl (3); Cerro Piedra China, San Angel Zurumucapio, 1700 masl (1); Presa del Cupatitzio, 1,600 masl (1).

Peromyscus gratus gratus Merriam, 1898. Specimens examined: Los Nogales, 1,750 masl (4); San Antonio Carapan, 2,000 masl (1); Bonilla, 2,100 masl (2); Cherán, 2,200 masl (2); Cerro Cicopién, 2,600 masl (21); Tzurumútaro, 2,100 masl, (1); San Lorenzo, 17 Km NE Uruapan, 1,900 masl (2); Cerro Comburindos, Tingambato, 2,350 masl (7); La Huizachera, Santa Rosa, 1,600 masl (2); Pátzcuaro, 2,500 masl (4).

Peromyscus levipes levipes Merriam, 1898. Specimens examined: Cerro Los Gallineros, 2.5 Km SW Angahuan, 2,400 masl (5); 6 Km N, 15 Km E Uruapan, 1850 masl (1); Presa del Cupatitzio, 1,600 masl (3); Ejido San Francisco, Uruapan, 1,600 masl (2); La Huizachera, Santa Rosa, 1,600 masl (1); Pátzcuaro, 2,500 masl (3).

Peromyscus maniculatus labecula Elliot, 1903. Specimens examined: Paracho, 2,750 masl (13); Cerro del Banco, Capacuaro, 2,350 masl (1); Cerro Las Cocinas, 2,250 masl (1); 8 Km N Capacuaro, 2,600 masl (14); Cerro Los Gallineros, 2 Km SE Angahuan, 2,450 masl (14); Barranca del Cupatitzio, 1,640 masl (1); La Huizachera, Santa Rosa, 1,600 masl (5); Pátzcuaro, 2,500 masl (3); $12 \mathrm{Km} \mathrm{N}$ Villa Escalante, 2,500 masl (2).

Peromyscus melanotis J. A. Allen y Chapman, 1897. Specimens examined: 6 Km SE Paracho, 2,800 masl (1); Cerro de la Bandera, Comachuén, 2,900 masl (1); La Huizachera, Santa Rosa, 1,600 masl (3); Pátzcuaro, 2,500 masl (2).

Peromyscus melanophys zamorae Osgood, 1904. Specimens examined: 4 Km SE Zamora, 1,800 masl (4); $5 \mathrm{Km}$ SE Zamora, 1800 masl (3).

Peromyscus spicilegus J. A. Allen, 1897. Specimens examined: Tanaco, 2,200 masl (1); La Huizachera, Santa Rosa, 1,600 masl (2); Presa del Cupatitzio, 1,600 masl (2).

Reithrodontomys chrysopsis chrysopsis Merriam, 1,900. Specimens examined: Cerro Cicopién, 2,600 masl (2); Sevina, 2,600 masl (12); El Abrojal, Opopeo, 2,100 masl (3); San Juan Nuevo, 1,850 masl (5).

Reithrodontomys megalotis saturatus J. A. Allen y Chapman, 1897. Specimens examined: San Antonio Carapan, 2,000 masl (1); Cerro Tres Esquinas, Cherán, 2,200 masl (1); Cerro San Marcos, 2,900 masl (1); Sevina, 2,600 masl (14); Cerro del Banco, Capacuaro, 2,350 masl (2); Cerro Cicopién, 2,600 masl (2); Bonilla, 2,100 masl (12); Cerro del Timbén, Mabusco, 2,100 masl (2); San Lorenzo, 17 Km NE Uruapan, 1,900 masl (9); Cerro Comburindos, Tingambato, 2,350 masl (1); Cerro Las Cocinas, 2,250 masl (3); Tancítaro (4); El Abrojal, 
Opopeo, 2,100 masl (7); San Juan Nuevo, 1,850 masl (3); Pátzcuaro, 2,500 masl (2); 2 Km W Cruz Gorda, 3100 masl (3); Cerro Piedra China, San Angel Zurumucapio, 1700 masl (1); Los Barrancos, Cutzato, 1700 masl (1).

Appendix 2. Continuación...

Reithrodontomys sumichrasti nerterus Merriam, 1901. Specimens examined: Los Nogales, 1,750 masl (1); Tanaco, 2,200 masl (1); Cerro Cicopién, 2,600 masl (7); Cerro del Banco, Capacuaro, 2,350 masl (3); Cerro San Marcos, 2,900 masl (5); El Abrojal, Opopeo, 2,100 masl (10); Paracho, 2,750 masl (1); Bonilla, 2,100 masl (3); Montelongos, 2,000 masl (3); 9 Km SW Pátzcuaro, 1,900 masl (1); Pátzcuaro, 2,500 masl (5); Cerro Comburindos, Tingambato, 2,350 masl (1); Cerro Las Cocinas, 2,250 masl (4); Sevina, 2,600 masl (1); 2 Km SE Angahuan, 2,450 masl (1); Cerro Los Gallineros, Angahuan, 2,400 masl (3); San Juan Nuevo, 1,850 masl (6); San Lorenzo, $17 \mathrm{Km}$ NE Uruapan, 1,900 masl (3); $1 \mathrm{Km} \mathrm{N}$, $2 \mathrm{Km}$ W Tancítaro, 2,900 masl (1). Barranca del Cupatitzio, 1,640 masl (3).

Sigmodon alleni Bailey, 1902. Specimens examined: Bonilla, 2,100 masl (1); San Lorenzo, 17 Km NE Uruapan, 1,900 masl (1); Barranca del Cupatitzio, 1,640 masl (1).

Sigmodon hispidus berlandieri Baird, 1855. Specimens examined: Cerro Cicopién, 2,600 masl (1); San Lorenzo, $17 \mathrm{Km}$ NE Uruapan, 1,900 masl (2); Cerro Las Cocinas, 2,250 masl (12); 2.5 Km SW Angahuan, 2290 masl (3); La Huizachera, Santa Rosa, 1,600 masl (3); Barranca del Cupatitzio, 1,640 masl (4);

Sigmodon mascotensis J. A. Allen, 1897. Specimens examined: Barranca del Cupatitzio, 1,640 masl (1); Cerro Piedra China, San Angel Zurumucapio, 1700 masl (4); Pátzcuaro, 2,500 masl (1).

Sylvilagus floridanus orizabae (Merriam, 1893). Specimens examined: 4 Km SW Uruapan, 1760 masl (1). 